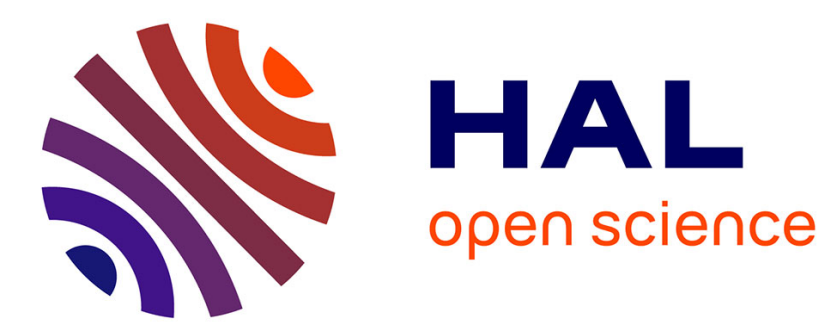

\title{
DES CONCESSIONS AUTOMOBILES EN QUÊTE D'UNE STRATÉGIE MARKETING
}

\author{
Carole Donada, Olivier Vidal
}

\section{To cite this version:}

Carole Donada, Olivier Vidal. DES CONCESSIONS AUTOMOBILES EN QUÊTE D'UNE STRATÉGIE MARKETING. Décisions Marketing, 2001, 23, pp.64-73. hal-00842007

\section{HAL Id: hal-00842007 https://hal.science/hal-00842007}

Submitted on 30 Aug 2013

HAL is a multi-disciplinary open access archive for the deposit and dissemination of scientific research documents, whether they are published or not. The documents may come from teaching and research institutions in France or abroad, or from public or private research centers.
L'archive ouverte pluridisciplinaire HAL, est destinée au dépôt et à la diffusion de documents scientifiques de niveau recherche, publiés ou non, émanant des établissements d'enseignement et de recherche français ou étrangers, des laboratoires publics ou privés. 


\section{DES CONCESSIONS AUTOMOBILES EN QUÊTE D'UNE STRATÉGIE MARKETING}

La distribution d'automobiles neuves en Europe est régie par un règlement d'exemption aux règles de la concurrence qui protège les concessionnaires. Ce règlement arrive à échéance et sa reconduction est aprement souhaitée par les concessionnaires qui risquent de perdre leur position d'acteurs incontournables du marché. Cette menace est amplifiée par l'arrivée de nouveaux entrants (grande distribution et acteurs du e-business) qui affinent leur stratégie marketing avant de pénétrer en force le marché. Dans ce contexte, les concessionnaires doivent repenser leur métier de distributeurs automobiles pour transformer les menaces en opportunités.

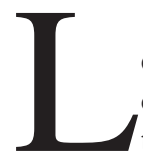

es spéculations sur l'avenir du système de la distribution automobile en Europe vont bon train. Alertés par la création puis l'échec des premiers hypermarchés de l'automobile aux ÉtatsUnis (AutoNation, CarMax, AutoCollection), les distributeurs européens s'inquiètent des changements possibles de leur profession. Par ailleurs, le règlement d'exemption aux règles de la libre concurrence qui assure aux concessionnaires leur statut de distributeurs exclusifs prend fin en 2002. Malgré cela, on assiste déjà aux tentatives très remarquées de la grande distribution qui se lance dans la vente de véhicules neufs à des prix plus que compétitifs. De plus, l'engouement des consommateurs pour les achats en ligne devient spectaculaire. En 1999, 0,5\% des ventes de véhicules neufs (VN) aux États-Unis étaient effectuées par Internet. Ces ventes représenteront probablement $1,5 \%$ du marché VN fin 2000, 4\% en 2001, 9\% en 2002 et, si ce rythme se poursuit dans les mêmes conditions, on achetera dès 2005 plus de VN en ligne que dans les concessions.

\author{
CAROLE DONADA et OLIVIER VIDAL
}

Dans ce contexte, le discours des concessionnaires français est unanime sur la menace que représente l'arrivée de la grande distribution pour leur métier. Il est en revanche plus partagé sur l'impact réel du développement des ventes en ligne. Les concessionnaires les plus modérés pensent que ce phénomène aura un effet marginal : "Internet, ne changera rien, les gens auront toujours besoin de venir chez nous pour acheter leur voiture", "acheter une voiture par Internet, c'est du non-sens, on ne risque rien". Au pire, "les concessionnaires suivront les constructeurs comme ils l'ont toujours fait". D'autres, plus nombreux et plus ouverts aux nouvelles technologies, profitent déjà de la situation : $21 \%$ d'entre eux ont en projet un site permettant la prise de commande en direct et $28,2 \%$ ont en projet un site dédié à la promotion (in BC Infos, oct 2000). Enfin, les plus pessimistes, mais aussi les moins nombreux pensent que "le métier de concessionnaire est de plus en plus difficile; avec Internet et les autres distributeurs, ce n'est plus la peine".

Carole Donada est Professeur adjoint à l'ESSEC au département Stratégie et Management. Ses recherches portent principalement sur l'industrie automobile, les coopérations interentreprises et les frontières d'entreprises.

Olivier Vidal est Professeur agrégé d'économie-gestion. Il a réalisé une étude comptable et financière sur la rentabilité des concessionnaires automobiles pour les professionnels du secteur. Il est également spécialiste du marché de l'optique. 
Ces divergences de perceptions des concessionnaires révèlent en fait des inquiétudes plus profondes dont l'origine n'est pas directement liée à la simple menace du e-commerce. Ces inquiétudes portent, d'une part, sur la relative dégradation de la situation économique de la profession et, d'autre part, sur la remise en cause de la légitimité de la mission des concessionnaires aux yeux des constructeurs. Depuis les années 1990, on a pu en effet assister à un effritement des marges des distributeurs ainsi qu'à une nette diminution du nombre de concessions rapporté au nombre de marques présentes sur le territoire. Dans ce contexte de marché de renouvellement où chaque vente gagnée par un distributeur correspond à peu près à une vente perdue pour un autre, nous pensons, comme ce spécialiste de la vente de voitures en ligne, qu'il y a "quand même matière à réinventer la distribution et à revivre une époque de pionniers qui a si longtemps fait le sel de ce métier?"1.

L'objectif de cet article est de montrer que les concessionnaires automobiles pourront "réinventer" leur métier s'ils développent une véritable stratégie marketing, c'est à dire, s'ils révisent leur modèle concurrentiel, redéfinissent leur portefeuille de produits et recherchent de nouvelles compétences. Notre proposition est que les menaces qui pèsent sur le système actuel peuvent être transformées en source d'opportunités : amélioration de la rentabilité de concessionnaires, accroissement des parts de marchés, et modification des relations avec les constructeurs. Pour argumenter cette proposition, nous nous sommes appuyés sur les résultats d'une analyse comptable et financière d'un concessionnaire type. A partir d'un budget représentatif d'un concessionnaire français moyen à la fin des années $90^{2}$, nous avons détaillé sa structure financière pour définir son seuil de rentabilité et sa structure de coûts. Une série d'entretiens réalisés auprès de concessionnaires et d'experts du secteur nous a permis de valider nos hypothèses de répartition de coûts et de comprendre le métier de concessionnaire. Cette étude nous a permis ensuite de justifier les axes stratégiques à développer.

L'article est structuré en trois parties. La première partie expose l'état des lieux économique, juridique et concurrentiel du système de distribution d'automobiles en Europe et tout particulièrement en France. La seconde partie reproduit la "photographie" comptable et financière de notre concessionnaire type. Enfin, la troisième et dernière partie de l'article fait la synthèse de ces éléments afin de proposer une stratégie marketing.

\section{$L^{\prime}$ état des lieux}

\section{Les principes de base}

La concession automobile est un maillon stratégique de la filière automobile parce qu'elle écoule la moitié des véhicules neufs mis en vente par les constructeurs tout en assurant le service après-vente et la distribution de pièces détachées pour les véhicules. Les constructeurs ne peuvent pas se passer d'un bon réseau de concessionnaires stable et étendu dont la qualité va déterminer :

- la représentation de la marque constructeur et sa notoriété;

- la couverture géographique des ventes et des parts de marché relatives sur le territoire;

- le maintien d'une rentabilité minimale sur tout le cycle de vie commercial des véhicules;

- le lissage des écarts entre la demande des clients et les capacités de production du constructeur;

- le retour direct d'informations au constructeur sur les niveaux de production à planifier, l'état et l'évolution des marchés, l'accueil des modèles par les clients.

L'origine du système de concessions remonte aux premières heures de la production automobile de masse aux USA. La répartition des tâches entre les acteurs est claire : les constructeurs produisent et les concessionnaires vendent. En Europe, la distinction est moins marquée. Aussi, les constructeurs allemands et français gardent le contrôle d'une partie de leurs ventes (à peu près 50\%) via un réseau de succursales ou filiales directes ; le reste étant proposé aux concessionnaires.

Deux grands principes fondateurs cadrent le système français de concessions : la sélectivité (le constructeur sélectionne ses concessionnaires) et l'exclusivité (seul le concessionnaire vend des véhicules neufs portant la marque du constructeur dont il est attaché). Les avantages de cette situation pour les concessionnaires sont doubles. Premièrement les concessionnaires disposent d'un droit préférentiel sur leur marché : chaque concessionnaire bénéficie notamment d'une assistance technique, commerciale, juridique et informatique délivrée par son constructeur. Deuxièmement, les concessionnaires sont assurés d'une rentabilité minimum négociée avec les constructeurs, compte tenu des investissements engagés et des ventes réalisées par chaque point de vente. En retour, les concessionnaires ont l'obligation de maintenir des stocks de véhicules et de pièces de 
rechange imposés par les constructeurs ; leur expansion géographique est limitée et leurs marges de manœuvre commerciales sont contraintes.

\section{Les évolutions récentes}

Depuis plus de 20 ans, les constructeurs ont cherché à diminuer leurs coûts en amont : reverse marketing, design to cost, production en flux tendus etc. Ils se tournent aujourd'hui vers la réduction des coûts en aval dont le total pèse à peu près pour $30 \%$ sur le prix de vente HT d'un véhicule. Première conséquence, on assiste à une diminution du nombre de concessions par marque. Pour illustrer, Opel va réorganiser d'ici la fin de l'année son réseau autour de 500 " circonscriptions de responsabilité " au lieu de 1000 distributeurs aujourd'hui en Allemagne. Cette nouvelle donne économique pour les concessionnaires est renforcée par deux principaux changements : la fin d'un cadre juridique européen favorable aux concessionnaires et l'entrée de nouveaux acteurs sur le marché.

\section{La fin d'un cadre juridique européen favorable aux concessionnaires}

Depuis le Traité de Rome, les deux principes de sélectivité et d'exclusivité propres au système de concession adopté en France ne sont pas en accord avec les dispositions juridiques de l'article 85-1 qui offre à tout opérateur économique européen une liberté de choix sur les marchés concernant les destinataires de ses offres et de ses ventes. C'est pourquoi, une exemption a rapidement été accordée aux concessionnaires automobiles. Cette clause d'exemption (révisée et assouplie en 1995 par le règlement 14975/95), permettait aux constructeurs et aux distributeurs d'automobiles d'inclure dans leurs accords commerciaux des clauses dérogeant à la libre concurrence, telles que les clauses d'exclusivité ou la clause interdisant aux concessionnaires de revendre des véhicules neufs à des revendeurs non agréés ${ }^{3}$. Les arguments développés par les concessionnaires pour justifier cette entorse au Traité de Rome étaient que "l'automobile est un produit complexe, un investissement lourd que le consommateur doit pouvoir choisir en connaissance de cause. Le consommateur a besoin de pouvoir comparer plusieurs modèles, les essayer et bénéficier des conseils d'un professionnel formé par le constructeur. Mais surtout, l'existence de réseaux lui garantit la pérennité d'un véritable service après-vente" ${ }^{4}$. La reconduction de ce règlement d'exemption est envisagée pour 2002 et les actions de lobbying des différents acteurs du secteur sont actives. D'un coté, il faut répondre au besoin croissant d'ouverture des frontières, d'harmonisation progressive des prix entre les différents pays européens et d'une plus grande concurrence entre les marques. De l'autre, il faut maintenir une profession et enfin, il faut protéger des consommateurs qui achètent un produit cher, complexe et dangereux s'il n'est pas adapté. En position de veille, les futurs entrants attendent le verdict et testent déjà quelques actions stratégiques avant de pénétrer en force sur le marché.

\section{L'arrivée de nouveaux acteurs sur le marché}

Parmi ces entrants potentiels, les premiers intéressés sont la grande distribution et les centrales d'achat. Maîtrisant parfaitement les logiques de domination par les coûts et les techniques de négociation avec les fournisseurs, ces nouveaux acteurs peuvent limiter les marges de manœuvre des concessionnaires et porter atteinte à leurs performances. Toutefois, les essais très médiatisés de la grande distribution via les magasins Auchan, Casino et Carrefour n'ont pas été transformés. Les concessionnaires se réjouissent de ces échecs relatifs et se persuadent sur le fait qu'ils sont aujourd'hui les seuls à pouvoir vendre une voiture et assurer les services dérivés. Toutefois, la menace reste bien réelle à en croire les titres d'articles d'Auto Infos ("Carrefour encore dangereux" ou "GMS/Réseaux : un face à face stratégique"6, ou encore cette proposition de Louis Schweitzer" ${ }^{7}$ si un groupe de distribution veut devenir concessionnaire Renault, en adoptant nos standards, nous n'y sommes pas opposés ". On comprend alors pourquoi $71,4 \%$ des concessionnaires attendent que leur organisation de tutelle les défende contre la grande distribution (in BC Infos, oct 2000).

L'arrivée des acteurs "virtuels" constitue une menace encore plus grande que celle exercée par la grande distribution. Aux États-Unis, la situation est jugée tellement préoccupante pour les concessionnaires que la très grande majorité des états ont redéfini leur cadre législatif pour protéger les vendeurs de véhicules neufs. Au Texas, par exemple, la loi HB3092 interdit formellement à tout acteur économique, autre qu'un distributeur reconnu et franchisé, de vendre un véhicule neuf dans l'état. Parmi ces nouveaux acteurs virtuels, on trouve d'un coté des distributeurs spécialisés dans l'automobile (voir encadré 1) et, de l'autre coté, des constructeurs qui testent de nouvelles formes de distribution en s'appuyant sur le potentiel de la toile informatique mondiale. Après la lean production qui a bouleversé l'amont de la filière constructeur, c'est au tour de la lean distribution de menacer les concessionnaires. Même si les constructeurs ne sont pas encore capables de proposer une organisation en Built to Order équivalente à celle de DELL, les expériences de MCC Smart sont riches d'enseignement pour la profession. 


\section{Encadré 1 : Les distributeurs US de VN sur Internet}

Deux grandes familles de distributeurs virtuels de véhicules neufs se partagent le marché de la e-distribution automobile américaine : les Referral Companies et les Direct e-Tailers.

Les Referral Companies (autobytel.com, AutoWeb.com...) sont des intermédiaires. Ce sont des sites de consultation qui améliorent la transparence de l'offre. Les internautes choisissent leurs véhicules et le site les dirige automatiquement vers le concessionnaire affilié le plus proche disposant du véhicule. La concurrence entre les concessionnaires est accentuée mais ces derniers assurent leur potentiel de marge en vendant directement au client final. Le plus connu et le plus actif de ces intermédiaires est autobytel (50\% du marché, 5000 concessionnaires affiliées, plus de 5 millions de visiteurs et plus de 50.000 ventes par mois). Son fondateur, Peter Ellis était un concessionnaire en faillite au début des années 90. Son idée de départ était de fournir en quelques heures le véhicule souhaité par le client et lui offrir un service totalement gratuit. La rémunération du site provient des droits d'accès payés par les concessionnaires affiliés. Ces droits varient entre 500 et 7500 dollars par mois.

Les Direct e-Tailers (DriceOff.com, Carsdirect.com...) sont des vendeurs de voitures directement par Internet. Ils se procurent leurs véhicules chez des concessionnaires ou directement auprès des constructeurs ou encore auprès de mandataires spécialisés dans les importations. Lorsqu'un concessionnaire fournit un e-Tailer, il ne perd pas le marché mais sa marge est inférieure à celle qu'il applique généralement sur un client particulier. Carsdirect.com appartient à cette catégorie. Ce site a été lancé en 1998 sur le principe de vente directe popularisé par Michael Dell (principal apporteur de capitaux au site). Jusqu'à l'automne 2000, CarOrder.com était un e-Tailers actif mais le site a fermé faute de trouver une rentabilité acceptable. L'expérience a couté quelques 100 millions de $\$$ à son actionnaire principal!

Quand on sait que le constructeur Ford Europe, qui connaît en ce moment de graves difficultés, gagne seulement 100 francs par véhicule vendu, on comprend sa tentation de revoir ses choix d'intégration verticale et ses potentiels de distribution de véhicules par internet. En France, les sites des constructeurs ne sont encore que des sites de présentation et de "teasing"7 mais la prospective sur les nouvelles formes de distribution est active.

\section{Photographie d'un concessionnaire type}

L'état des lieux qui vient d'être présenté laisse prévoir des changements majeurs pour les concessionnaires. Afin de comprendre l'impact possible de ces changements, il est nécessaire d'analyser la situation financière ainsi que le profil stratégique générique des concessionnaires. C'est pourquoi, nous avons "photographié " un concessionnaire type. Sa photo reproduit sa situation comptable et financière ainsi que son portefeuille d'activités. Un effet de zoom accentue plus particulièrement ses sources de rentabilité activité par activité, sa structure de coûts et sa marge incompressible globale. Pour réaliser cette photographie, nous sommes partis d'un budget type représentatif d'un concessionnaire français moyen à la fin des années 1990. Ce budget n'est pas nécessairement le reflet de la situation financière de n'importe quel concessionnaire. Il est une projection idéale avec un résultat reconnu comme satisfaisant par les experts du secteur. Pour interpréter les informations comptables, nous avons réalisé une série d'entretiens auprès de concessionnaires. Ces entretiens nous ont permis d'une part de comprendre la répartition des coûts fixes liés à la structure d'une concession, et la part des coûts variables liés au volume d'activité annuel et, d'autre part, de distinguer les coûts directs propres à chaque activité des coûts indirects qui pèsent sur l'ensemble de la concession,

\section{Zoom sur la structure de coûts}

Le travail du concessionnaire automobile repose sur quatre activités : la vente de véhicules neufs (VN), la vente de véhicules d'occasion (VO), la vente en magasin de pièces détachées (MPR), la réparation et le service après vente en atelier (A). Notre photographie met en évidence des seuils de rentabilité très différents selon les activités :

- L'activité $V N$, véritable raison d'être du concessionnaire, n'est paradoxalement pas nécessairement son activité la plus confortable. Même si les coûts fixes (CF) sont relativement faibles, les coûts variables (CV) sont élevés (c'est le concessionnaire qui achète le véhicule au constructeur) et la marge relative 


\section{Encadré 2 : Plus de détails sur la structure des coûts}

La situation est particulièrement difficile à supporter sur les activités VN et VO puisqu'une augmentation de $10 \%$ des frais fixes nécessite une augmentation équivalente des ventes pour les compenser. Dans notre simulation, un investissement de l'ordre de 200KF (ce qui est relativement modeste) suppose la vente de 100 véhicules neufs supplémentaires (ce qui esténorme). Par ailleurs, une diminution de 1 point de la marge sur coûts variables (c'est à dire de 1\% du prix de vente) a des conséquences très différentes selon les activités. Pour illustrer, il faut vendre $25 \%$ de VN en plus pour compenser une telle diminution (une remise de $1000 \mathrm{~F}$ par véhicule neuf doit être au moins compensée par la vente sur l'année de 125 véhicules supplémentaires). Dans l'activité VO, le phénomène est accentué ; il faut vendre $62 \%$ de VO en plus pour compenser une perte de 1 point de la marge sur coûts variables. En revanche, il faut simplement augmenter de 3,5\% le volume des ventes magasin et de 1,7\% le volume de l'activité atelier pour compenser une perte de 1 point de $\mathrm{M} / \mathrm{CV}$.

sur coût variable $(\mathrm{M} / \mathrm{CV})$ ne dépasse pas $5 \%$. Bien évidemment, cette faible marge relative, appliquée sur un prix de vente moyen de l'ordre de $100 \mathrm{KF}$, génère une marge en valeur très confortable. Cependant, toute légère augmentation des $\mathrm{CF}$ ou baisse de la M/CV diminue immédiatement le résultat de l'entreprise (voir encadré 2). Par ailleurs, le concessionnaire ne peut générer des résultats importants que si le seuil de rentabilité est très largement dépassé. En cas de conjoncture économique défavorable, une baisse, même légère, des ventes peut générer très vite des pertes.

- L'activité VO est encore plus difficile à maîtriser. Pour tirer profit de cette activité, notre concessionnaire type devrait vendre deux VO pour un VN. Les coûts variables sont élevés (l'achat du véhicule au revendeur est généralement un argument pour vendre un $\mathrm{VN}$ ), les coûts fixes restent relativement faibles et la marge sur coût variable (M/CV) est extrêmement faible (3\%). Ainsi, toute augmentation des CF ou diminution supplémentaire de la $\mathrm{M} / \mathrm{CV}$, même infime, a des conséquences lourdes sur le résultat de l'entreprise.

- L'activité atelier a des coûts fixes importants (outillage, locaux...), mais les coûts variables sont relativement faibles et la marge sur coût variable est élevée (60\%). Cette structure ne permet pas facilement le dépassement du seuil de rentabilité sur de faibles volumes et rend cette activité structurellement déficitaire. En revanche, tout dépassement du seuil génère un résultat immédiat et important dont le concessionnaire profite peu car, ne voulant pas ressembler à un "garage", il alloue peu de ressources à l'atelier qui, in fine, lui rapporte peu.

- L'activité vente de pièces de rechange en magasin (MPR) a une structure de coûts différente. Les coûts variables sont modérés, les coûts fixes sont relativement faibles, et la marge sur coût variable est satisfaisante (30\%). C'est l'activité la plus rentable, et surtout la moins risquée du concessionnaire, et cela d'autant plus que le niveau moyen des ventes de pièces de rechange est relativement peu sensible aux aléas conjoncturels.

Ce zoom comptable et financier nous permet de faire deux constats. Premièrement, le concessionnaire est relativement fragile sur son activité de base car il est très sensible aux aléas de la conjoncture économique. Deuxièmement, une baisse de la marge commerciale (rabais accordés aux clients, pression du constructeur...) ou une augmentation de ses frais fixes (formation du personnel, modernisation de l'outillage lié aux progrès technologiques, ...) même faible en valeur relative, entraîne des conséquences lourdes sur ses résultats.

\section{Zoom sur le portefeuille d'activités}

La représentation graphique des activités de notre concessionnaire type montre un portefeuille nettement déséquilibré et peu dynamique. $80 \%$ du CA provient de la vente de véhicules et $20 \%$ seulement des activités atelier et magasin. La performance globale du concessionnaire dépend donc directement de sa capacité à faire du volume sur des produits dont la marge relative est faible. On observe que l'activité VN est une activité " vache à lait " qui est peu productive puisque, bien que les besoins de financement soient faibles, les marges le sont aussi et les perspectives de croissance sur ce marché mature correspondent à peine au taux de renouvellement des véhicules. En outre, la performance de cette activité est directement liée à celles des ventes de VO : pour doper ses VN, le concessionnaire peut être tenté de reprendre l'ancien véhicule du client à un prix élevé, ce qui diminuera d'autant la rentabilité de son activité VO. En définitive, la contribution directe à la création de valeur de l'activité VO pour le concessionnaire est 
Schéma 1 : Représentation graphique du portefeuille d'activités du concessionnaire

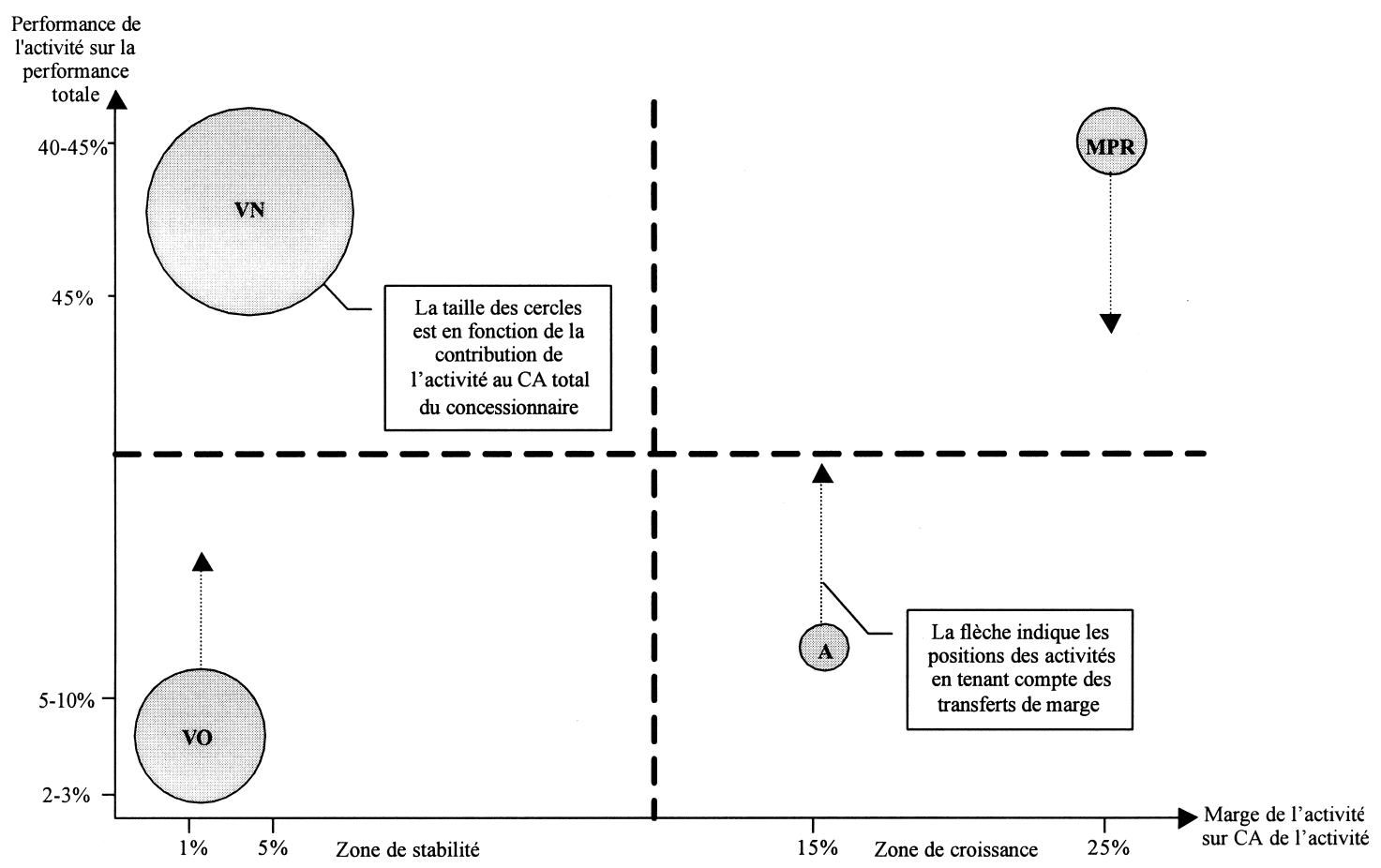

très faible, voire nulle. Ce sont donc paradoxalement les activités MPR et Atelier qui paraissent les plus dynamiques dans le portefeuille stratégique du concessionnaire et qui offrent des potentiels de croissance théorique intéressants. Cependant, ces activités, supports de la vente de véhicules, ne représentent qu'une faible part du chiffre d'affaires du concessionnaire et, bien que l'activité MPR contribue fortement à sa performance, le volume d'affaires reste trop faible pour rééquilibrer le portefeuille. De son coté, l'atelier a de meilleures perspectives de croissance mais son volume d'activité et sa contribution à la performance de l'entreprise sont trop faibles.

Au final, cette photographie met en évidence l'importance d'une double contrainte qui pèse sur les concessionnaires : une contrainte sur les prix et une contrainte sur les coûts. La contrainte sur les prix est liée, d'une part, aux constructeurs qui limitent la marge de manœuvre des distributeurs en fixant les prix de vente catalogue des véhicules et, d'autre part, au nouveau pouvoir de négociation des clients qui réclament des remises de prix de plus en plus importantes. Dans ce contexte, les actions des acteurs indirects tels que les établissements financiers qui proposent des crédits, les organismes d'assurance qui fixent le montant des primes ou les administrations publiques qui proposent des remises "gouvernementales", accentuent d'autant les effets de cette première contrainte sur les prix. La contrainte sur les coûts, quant à elle, dépend des constructeurs qui déterminent les prix d'achat des véhicules neufs et le montant des primes, des clients qui négocient le prix d'achat des véhicules d'occasion, des fournisseurs d'équipements et de matériels de plus en plus sophistiqués pour les concessionnaires ou des administrations qui imposent des normes techniques particulières. Cette contrainte est renforcée par la contraction des délais de livraison des véhicules aux clients ainsi que par une demande de qualité de service toujours plus exigeante de la part des constructeurs et des clients (formation supplémentaire pour le personnel, nouvelles procédures de travail, audits à effectuer...). Dans ce contexte, notre concessionnaire doit saisir les nouvelles opportunités de marché qui se présentent et repenser son métier de distributeur.

\section{Transformer les menaces en opportunités}

Pour transformer les menaces des nouvelles formes de distribution en opportunités, il faut remettre à plat sa stratégie marketing. Jusqu'à présent, la stratégie la 
plus recherchée par les constructeurs et la plus suivie par les concessionnaires est celle du volume. La poursuite de cette stratégie a-elle encore du sens?

\section{Stratégie de volume, la fin d'une époque}

La stratégie de volume est bien adaptée lorsque les coûts fixes sont élevés puisqu'on peut les répartir sur des quantités importantes. Malheureusement, nous avons vu que les coûts fixes des concessionnaires sur les VN sont relativement faibles et que les coûts variables sont élevés (achats de véhicules au constructeur). Cette situation oblige donc les concessionnaires à dépenser beaucoup d'énergie pour négocier auprès des constructeurs le maintien de leurs marges afin de maintenir leur performance économique. Les marges brutes actuellement pratiquées oscillent entre 13 et $15 \%$, auxquelles peuvent s'ajouter les primes constructeurs d'incitations à la vente (entre $0 \%$ et $4 \%$ ). Or, un concessionnaire qui vend 500 VN (moyenne nationale) a une rentabilité satisfaisante si sa marge brute est supérieure ou égale à 18,7\%. Autrement dit, le concessionnaire qui n'atteint pas les objectifs de ventes fixés pour l'obtention des primes constructeurs ne peut assurer longtemps sa pérennité. Pour y parvenir, il peut rechercher la croissance en augmentant la taille de sa concession. Cette option suppose bien évidemment une structure financière importante et une situation compétitive suffisamment favorable pour que les concurrents les plus proches ne se lancent pas dans la même politique. Il faut aussi que le constructeur n'ajuste pas directement le niveau des primes en fonction de la nouvelle taille de la concession.

Dans ce cadre, de plus en plus de concessionnaires élargissent leur marge de manouvre en s'appuyant sur le e-commerce. Ils créent leur propre site, participent à des portails et à des sites collectifs de concessionnaires ou s'impliquent dans des sites d'achat multi-produits. Ces concessionnaires réalisent en moyenne 10 à 15\% de leur chiffre d'affaires grâce à internet. Toutefois, ce chiffre d'affaires gagné sur la toile ne correspond pas forcément à une réelle croissance de l'activité ; c'est plutôt une autre façon de vendre. De plus, la croissance des ventes par internet ne garantie pas la performance puisqu'elle renforce significativement la contrainte prix. Comme chacun sait, les internautes recherchent le prix le plus bas et ils se servent de la toile mondiale pour inciter à la transparence des marchés. JD Power \& Associates estiment que $65 \%$ des achats de véhicules aux États-Unis aujourd'hui sont préparés en ligne pour pouvoir négocier des prix moyens plus faibles de $500 \$$ par rapport aux prix négociés directement en concession.
Cette pression des clients sur les prix, et donc sur les marges des concessionnaires, est facilitée par les intermédiaires du Web qui communiquent majoritairement sur leur capacité à proposer les prix les plus bas (" notre équipe de conseillers recherche pour vous gratuitement votre véhicule en effectuant un appel d'offres auprès des concessionnaires sur tout le marché français" cf. site d'Autovalley). Ainsi, Peter Ellis, fondateur d'Autobytel, ne cesse de répéter que le client doit savoir à quel prix le concessionnaire a acheté la voiture au constructeur. Enfin, les internautes s'associent de plus en plus à des opérations d'achats groupés (group buying) pour obtenir des prix de détail équivalents aux prix de gros. Ces nouvelles pratiques mettent en évidence les limites de la stratégie de distribution push qui domine le secteur depuis près d'un siècle. Le système qui consistait, pour les constructeurs, à définir des produits dans leurs bureaux de Detroit, de Tokyo, de Paris ou de Turin, puis à les vendre à prix fixe à leurs distributeurs, vit probablement ses dernières heures. Constructeurs et distributeurs doivent revoir leur organisation parce que les consommateurs les y obligent. Il semble donc difficile de tout miser sur le e-commerce pour assurer une stratégie de volume rentable et défendable dans le temps sans remettre en cause le système actuel de la distribution automobile dans les concessions. Dernière menace, si l'euphorie des internautes pour acheter en ligne une voiture se maintient, les constructeurs peuvent être tentés de se séparer de leurs concessionnaires pour distribuer eux-mêmes leurs véhicules. BuyPower (GM), Get a quote (Chrysler) ou Buyerconnection (Ford) sont bien des prototypes de futurs sites de vente directe en ligne des constructeurs américains.

Conscients de ces risques, certains concessionnaires tirent la sonnette d'alarme. Leur première action est de s'adresser directement aux constructeurs auxquels ils réclament un potentiel de marge plus important. Il nous semble que les gains de cette requête seront de très court terme car, plus la marge des distributeurs sera perçue comme importante, plus les constructeurs seront tentés de vendre directement leurs produits, et plus les groupes d'acheteurs exerceront des pressions fortes pour diminuer le prix public. La seconde action des concessionnaires porte sur des opérations de lobbying auprès des autorités politiques européennes, via le CNPA (Conseil National des Professions de l'Automobile). Les propositions visent à protéger la profession en réclamant notamment la reconduction du règlement d'exemption 1475/95, l'annualisation du contrôle technique des véhicules, le renforcement des contrôles anti-pollution, la diminution de la fiscalité sur l'automobile ou encore, le développement d'une 
politique qui favorise le choix de l'automobile comme moyen de transport. Si ces revendications aboutissent, les actions des lobbies pourront sans doute aider les concessionnaires. Mais à nouveau, notre diagnostic laisse penser que la victoire sera de courte durée si les nouvelles tendances du marché se confirment. La priorité stratégique n'est pas défensive mais attaquante et largement tournée vers le marketing stratégique.

\section{L'occasion d'une nouvelle stratégie basée sur un marketing stratégique relationnel}

Selon Porter (1), l'alternative logique à la stratégie de volume est la stratégie de différenciation : différenciation sur le produit et différenciation sur le service. La différenciation sur le produit est très difficile à mettre en œuvre pour les concessionnaires qui doivent écouler la production décidée par leur constructeur. Ils vendent essentiellement les véhicules qu'ils ont en stock et, sauf exception, l'acheteur trouve rarement l'exact produit qu'il souhaite (choix restreint sur les séries, les couleurs, les options...). Déçu, l'acheteur va accepter un autre modèle en échange d'équipements supplémentaires ou différents de ceux proposés initialement, et de remises de prix conséquentes. Cette situation, où le concessionnaire est davantage un vendeur qu'un médiateur entre les demandes des consommateurs et les offres des constructeurs, rend impossible toute différenciation sur le produit et oblige le distributeur à se différencier sur le service. Cette deuxième option est également très difficile à mettre en œuvre car elle nécessite des investissements particuliers qui entraînent un accroissement rapide des coûts fixes (coûts de formation ou de requalification de la force de vente, budget publicitaire accru, études marketing approfondies, achats de nouveaux équipements qui valoriseront la perception du client sur la qualité des prestations offertes etc.). En outre, le concessionnaire est dépendant de l'image de la marque qu'il distribue. Dans ce cadre, la marge de manœuvre des concessionnaires est particulièrement étroite. La réussite d'une stratégie de différenciation nécessite une redéfinition complète du métier de concessionnaire et ne peut se faire sans une coopération accrue avec les constructeurs.

Au niveau marketing, cela doit se traduire par l'abandon d'un marketing transactionnel au profit d'un marketing relationnel (2-4). Le concessionnaire " type" est d'un coté menacé sur ses volumes et, de l'autre, il ne peut augmenter sa rentabilité en élevant ses marges. Ce dilemme classique du distributeur "enlisé dans la voie médiane" (1) peut être résolu par l'amélioration des relations client-concessionnaire et concessionnaire-constructeur (3-4).

L'amélioration des relations client-concessionnaire passe par une plus grande confiance dans la relation commerciale et permet une fidélisation très forte du client. Pour y parvenir, le concessionnaire doit déconstruire sa chaîne de valeur (5) et recomposer son portefeuille d'activités. Jusqu'à présent, pour vendre des voitures neuves (sa principale activité), le distributeur doit assurer la reprise des véhicules d'occasion, offrir le service après vente, la réparation et l'entretien dans son atelier et enfin, vendre des pièces détachées. Il doit aussi proposer des solutions de financement et d'assurance en partenariat avec des courtiers spécialisés ou les filiales financières des constructeurs (Sovac...). C'est la bonne maîtrise de cette chaîne par le concessionnaire qui assurait sa rentabilité. Depuis peu, cette mécanique est menacée de toutes parts : le client commence par vendre seul son ancien véhicule directement sur internet, il commande en ligne sa nouvelle voiture après avoir recherché le prix le plus faible et les garanties les plus étendues, il négocie lui-même une solution de financement et d'assurance avec l'intermédiaire qu'il choisit, il s'adresse ensuite à des réparateurs franchisés et spécialistes pour assurer les tâches d'entretiens courantes et obligatoires (les freins chez Midas, les pots d'échapement chez Speedy...), il achète ses pièces détachées dans les grandes surfaces (Leclerc Auto, Carrefour...) etc. Face à cette nouvelle donne, le concessionnaire est fragilisé. Il lui faut reconquérir la relation de confiance qu'il avait jusqu'alors avec son client et mettre en place un véritable marketing relationnel (6).

L'amélioration des relations concessionnaireconstructeur est indispensable pour y parvenir, et ne peut se faire sans créer une relation partenariale de type gagnant-gagnant. Ce partenariat favorise la mise en place de la "lean distribution" et, avec elle l'organisation marketing en "One to One". Sans cette organisation qui repose notamment sur la qualité des plateformes électroniques $B$ to $B$ et $B$ to $C$ dans le secteur, toute action de différenciation produit sera vaine et toute différenciation sur le service sera coûteuse et difficile à maintenir. En revanche, une fois le système mis en place, les commandes des clients seront directement transmises au constructeur et à l'ensemble des fournisseurs d'équipements ; le juste à temps permettra de diminuer les dépenses de stockage qui représentent la plus grande part des frais de distribution. De leur coté, les concessionnaires libérés des contraintes de stocks, pourront proposer et facturer des services supplémentaires qui ne seront pas simplement la compensation des insatisfactions 
du client. Ils quitteront leur " rôle de transmetteurs d'ordre, d'officiants zélés de la grand'messe stratégique décidée dans les bureaux de leur concédant" (in, BC Info, octobre 2000), pour retrouver un rôle d'intermédiaire qui permettra de réorganiser le portefeuille du concessionnaire autour de la notion de "service automobile" : totalité des services liés à l'après achat, location de véhicules occasionnels et autres formes d'accès à l'utilisation d'une automobile, etc.

\section{Conclusion}

En conclusion, le secteur de la distribution automobile connaît des changements importants qui menacent la position occupée par les concessionnaires depuis des dizaines d'années. Ces changements naissent de la structure du marché automobile européen, de l'entrée d'acteurs qui bouleversent les règles du jeu et des comportements des nouveaux consommateurs plus informés, plus volatiles et plus exigeants. Dans ce contexte, les concessionnaires sont d'autant plus fragilisés que la composition de leur portefeuille d'activités et leur structure de coûts ne leur laissent que peu d'espace de liberté pour protéger leur avantage concurrentiel. Leur stratégie de négociation des marges auprès des constructeurs pour maintenir leur rentabilité n'est donc plus adaptée. Ils doivent réinventer leur métier en s'appuyant sur une véritable stratégie marketing (5-7). Dès lors, le développement du e-commerce devient une opportunité car toutes les actions qui améliorent les services offerts aux clients seront à privilégier. En amont, une bonne lecture de la toile électronique sera la base des opérations de segmentation et des analyses du comportement du consommateur tout au long de sa vie. Les coopérations récentes avec les géants des portails (Ford associé à Yahoo), les grands fournisseurs d'accès (GM et AOL) ou les sites généralistes de vente en ligne (Amazon.com qui dispose d'un portefeuille de 13 millions de clients et qui ouvre un site de commerce automobile), montrent combien les constructeurs veulent se rapprocher des consommateurs pour mieux connaître leurs profils, leurs cibles potentielles et devenir ainsi des " consumer company" (Les Echos, 11/01/2000). C'est en s'imposant comme médiateur incontournable que le concessionnaire pourra repositionner son portefeuille d'activités.
Notes

1 Extrait de l'éditorial de Jean Triomphe pour la revue du site www.procar.com 5.

2 Il s'agit d'une projection idéale avec un résultat comptable reconnu comme satisfaisant par les experts du secteur. Les détails de l'étude comptable et financière de ce concessionnaire type ont été publiés par le CNPA dans le document : "la rentabilité d'une concession automobile".

3 Cf. "Le droit de la distribution automobile : un bilan pour 1'avenir", C. Collard, La lettre du Gerpisa, n¹35

4 Position du CNPA exprimée à Bruxelles, in Auto Infos 1096, p. 4.

5 Auto Info 1096 p. 4

6 Auto Info 1095 p. 16

7 BC Info 20.

8 Le "teasing" consiste à attirer les internautes futurs clients sur des modèles non encore sortis. Volskwagen a présenté ainsi sa Beetle bien avant sa commercialisation, Renault et Peugeot font de même pour l'Avantime et la 607 .

\section{Bibliographie}

(1) Porter M.E. (1982) Choix stratégiques et concurrence, Economica, Paris.

(2) Grönros C. (1995) Relationship Marketing : The Strategy Continum, Journal of The Academy of Marketing Science, 23, $4,252-254$.

(3) Leforestier B. (1996) Le marketing relationnel des distributeurs, Décisions Marketing, 7, Janv-Avril, 47-52.

(4) Jap S.D. (1999) Pie-expansion efforts : Collaboraton processes in buyer-supplier relationships, Journal of Marketing Research, 36, 4, 461-475.

(5) Evans P., Wurster T.S. (2000) Net Stratégies, Editions d'Organisation, Paris.

(6) Perrien J. (1998) Le marketing relationnel : oui mais..., Décisions Marketing, 13, Janv-Avril, 85-88.

(7) Filser M. (1992) Gérer l'évolution de son système de distribution. Le cas de la distribution automobile. Revue Française de Gestion, septembre-octobre, 108-116. 